\title{
THE BOXDOT CONJECTURE AND THE GENERALIZED MCKINSEY AXIOM
}

\author{
CHRISTOPHER STEINSVOLD
}

\begin{abstract}
The Boxdot Conjecture is shown to hold for a novel class of modal systems. Each system in this class is $\mathrm{K}$ plus an instance of a natural generalization of the McKinsey axiom.
\end{abstract}

\section{The Conjecture}

In modal logic, the following translation, $t$, is the boxdot translation,

$$
\begin{aligned}
& t p=p \\
& t \perp=\perp \\
& t(\phi \rightarrow \psi)=(t \phi \rightarrow t \psi) \\
& t \square \phi=(\square t \phi \wedge t \phi)
\end{aligned}
$$

The name derives from the use of $₫ \phi$ as a symbol for $\square \phi \wedge \phi$ in Boolos [1]. We continue the use of this symbol, and also use $\diamond \phi$ for $\diamond \phi \vee \phi$.

Where $\mathrm{K}$ is the minimal normal modal logic, $\mathrm{K} \oplus \phi$ is the smallest normal modal logic containing $\phi$. KT is $\mathrm{K} \oplus \square \phi \rightarrow \phi$. In [3], French and Humberstone conjectured that, for all normal modal logics L:

$$
\begin{aligned}
& \text { if }(\forall \psi)(\mathrm{L} \vdash t \psi \text { if and only if } \mathrm{KT} \vdash \psi) \text {, } \\
& \text { then } \mathrm{L} \subseteq \mathrm{KT} \text {. }
\end{aligned}
$$

This is the Boxdot Conjecture. French and Humberstone laid groundwork for future discussion and showed that the conjecture holds for all $\mathrm{K} \oplus \phi$ with $\phi$ of modal degree 1. As the authors point out, it is not difficult to show the converse of the conjecture is true, and also not difficult to show the conjecture holds for any extension of KT, yet it seems there is no clear path toward dealing with all other cases of the conjecture. In Steinsvold [4], the conjecture was shown to hold for all $\mathrm{K} \oplus \mathrm{G}^{h i j k}$, where $h, i, j, k \in \mathbb{N}$, and

$$
\mathrm{G}^{h i j k}: \diamond^{h} \square^{i} p \rightarrow \square^{j} \diamond^{k} p
$$

We use $\mathrm{G}^{h i j k}$ as an arbitrary instance of this axiom schema (an instance of the schema is given by a specific $h, i, j, k)$. The ' $\mathrm{G}$ ' is for Geach. Here we show the conjecture holds for $\mathrm{K} \oplus \mathrm{M}^{l m n o}$ where $l, m, n, o \in \mathbb{N}$, and

$$
\mathrm{M}^{l m n o}: \square^{l} \diamond^{m} p \rightarrow \diamond^{n} \square^{o} p
$$

We use $\mathrm{M}^{l m n o}$ as an arbitrary instance of this axiom schema (an instance of the schema is given by a specific $l, m, n, o)$. The ' $\mathrm{M}$ ' is for McKinsey, as $\mathrm{M}^{1111}$ is the McKinsey axiom,

Australasian Journal of Logic (15:3) 2018, Article no. 2 


$$
\mathrm{M}: \square \diamond p \rightarrow \diamond \square p
$$

$\mathrm{KM}$ is $\mathrm{K} \oplus \mathrm{M}^{1111}$. See Goldblatt and Hodkinson [2] for more information on this axiom. As there are infinitely many $\mathrm{M}^{l m n o} \in \mathrm{KT}$, we show:

$$
\text { for all } \mathrm{M}^{l m n o} \notin \mathrm{KT},(\exists \psi)\left(\mathrm{K} \oplus \mathrm{M}^{l m n o} \vdash t \psi \text { and } \mathrm{KT} \nvdash \psi\right)
$$

This is our main result. The paper is organized as follows. We conclude this section with a description of our overall strategy. In Section 2 we use $\mathrm{KM}$ as an example to illustrate our method. In Section 3 we present various preliminary results. In Section 4, we present the models which will aid our strategy. The following sections deal with the essential cases, and we conclude with our main result in Section 7 .

A formula is a boxdot formula if it is the translation of some formula. We use $\phi^{\square}$ and $\psi^{\square}$ for arbitrary boxdot formulas.

The following definition is novel and we use it to explain our strategy. The definition is a generalization of the notion of a surrogate (from [4]).

Definition 1.1. Call $\phi^{\square} \rightarrow \psi^{\square}$ an exterpolant for $\alpha \rightarrow \beta$, if

$$
\begin{aligned}
& \text { (A) } \mathrm{K} \vdash \phi^{\square} \rightarrow \alpha \\
& \text { (B) } \mathrm{K} \vdash \beta \rightarrow \psi^{\square}
\end{aligned}
$$

It follows that an exterpolant for $\alpha \rightarrow \beta$ is a theorem of $\mathrm{K} \oplus \alpha \rightarrow \beta$. Call an exterpolant trivial if it is a theorem of K. Note that there is a single trivial exterpolant for every conditional $\alpha \rightarrow \beta$, namely $\perp \rightarrow \mathrm{T}$ (as $\mathrm{K} \vdash \perp \rightarrow \alpha$, $\mathrm{K} \vdash \beta \rightarrow \mathrm{T}, t \perp=\perp$, and $t \mathrm{~T}=\mathrm{T})$. Our interest here lies with non-trivial exterpolants. As for the name 'exterpolant' itself, the informal idea is that an exterpolant seems like the opposite of an interpolant. Conditions (A) and (B) of definition 1.1 are the informal justification for our use of the word 'exterpolant' (in loose contrast to an interpolant, $I$, for $C \rightarrow D$, where $C \rightarrow I$ and $I \rightarrow D$ are theorems)).

Our strategy is to construct exterpolants for each $\mathrm{M}^{\text {lmno }}(\notin \mathrm{KT})$ and then show these exterpolants are not theorems of K. Using the following Lemma, we can then conclude the conjecture holds for each $\mathrm{K} \oplus \mathrm{M}^{l m n o}(\nsubseteq \mathrm{KT})$.

Lemma 1.2. If $\mathrm{L} \vdash t \phi$ and $\mathrm{K} \vdash t \phi$, then $(\exists \gamma)(\mathrm{L} \vdash t \gamma$ and $\mathrm{KT} \nvdash \gamma)$.

Proof. Assume L $\vdash t \phi$ and $\mathrm{K} \vdash t \phi$. As mentioned in [3], for all $\psi$,

$$
\mathrm{K} \vdash t \psi \text { iff } \mathrm{KT} \vdash \psi
$$

Thus KT $\forall \phi$. Thus, $(\exists \gamma)(\mathrm{L} \vdash t \gamma$ and $\mathrm{KT} \nvdash \gamma)$

To utilize an example from [4], consider

$$
(\neg p \wedge \diamond p) \rightarrow[(q \rightarrow p) \rightarrow \odot(q \rightarrow p)]
$$

This sentence is an exterpolant for $\diamond p \rightarrow \square p$. The antecedent and consequent are boxdot formulas, and

$$
\begin{aligned}
& \text { (A) } \mathrm{K} \vdash(\neg p \wedge \diamond p) \rightarrow \diamond p \\
& \text { (B) } \mathrm{K} \vdash \square p \rightarrow[(q \rightarrow p) \rightarrow \odot(q \rightarrow p)]
\end{aligned}
$$


Furthermore,

(1) $\mathrm{K} \oplus \diamond p \rightarrow \square p \vdash(\neg p \wedge \odot p) \rightarrow[(q \rightarrow p) \rightarrow \odot(q \rightarrow p)]$

(2) $\mathrm{K} \nvdash(\neg p \wedge \diamond p) \rightarrow[(q \rightarrow p) \rightarrow \odot(q \rightarrow p)]$

Thus by Lemma 1.2, the Boxdot Conjecture holds for $\mathrm{K} \oplus \diamond p \rightarrow \square p$.

Our overall strategy is similar to that of [4], and the work there simplifies the work here, as there are infinitely many $\mathrm{M}^{l m n o}$ which are instances of $\mathrm{G}^{\text {hijk }}$. For instance, $\mathrm{M}^{1002}=\mathrm{G}^{0120}(=\square p \rightarrow \square \square p)$. Thus, our strategy is as follows. For each $\mathrm{M}^{l m n o} \notin \mathrm{KT}$, and also not an instance of some $\mathrm{G}^{\text {hijk}}$, we construct an exterpolant for $\mathrm{M}^{l m n o}$ which is not a theorem of $\mathrm{K}$, then apply Lemma 1.2 to $\mathrm{K} \oplus \mathrm{M}^{l m n o}$.

A frame $F$ is a pair $\langle W, R\rangle$ where $W$ is a non-empty set and $R \subseteq W \times W$. Members of $W$ are worlds or points. A valuation $V$ is a function from the set of propositional variables into the power set of $W . M=\langle W, R, V\rangle$ is a model. We define truth in a model at a world as follows:

$$
\begin{aligned}
& M, w \vDash p \text { iff } w \in V(p) \\
& M, w \vDash \perp \text { iff } 0=1 \\
& M, w \vDash \phi \rightarrow \psi \text { iff if } M, w \vDash \phi \text { then } M, w \vDash \psi \\
& M, w \vDash \square \phi \text { iff }(\forall x)(\text { if } w R x \text { then } M, x \vDash \phi)
\end{aligned}
$$

$\phi$ is valid in the model $M$ iff $\phi$ is true at every world in $M . \phi$ is valid in the frame $F$ iff $\phi$ is valid in every model based on $F$.

\section{2. $\mathrm{KM}$}

We use $\mathrm{KM}(\mathrm{K} \oplus \square \diamond p \rightarrow \diamond \square p)$ as an example. First, observe that $\diamond\left(p \wedge q_{1}\right) \wedge \neg q_{1}$ implies $\diamond p$. For $\diamond\left(p \wedge q_{1}\right)$ is $\diamond\left(p \wedge q_{1}\right) \vee\left(p \wedge q_{1}\right)$, and so if $\neg q_{1}$ is true, the disjunct $\left(p \wedge q_{1}\right)$ must be false. With this in mind consider the following theorem of $\mathrm{K}$,

$$
\text { (A) } \mathrm{K} \vdash \odot\left[\left(\diamond\left(p \wedge q_{1}\right) \wedge \neg q_{1}\right) \vee\left(\diamond\left(p \wedge q_{2}\right) \wedge \neg q_{2}\right)\right] \rightarrow \square \diamond p
$$

The antecedent implies (though is not equivalent to) the claim that at all possible worlds, either $p$ and $q_{1}$ are both possible or $p$ and $q_{2}$ are both possible, thus, either way, at all possible worlds $p$ is possible (the consequent). Significantly, the antecedent is a boxdot formula which implies the antecedent of the McKinsey axiom. Now, take the contraposition of the theorem of $\mathrm{K}$ in $(\mathrm{A})$, put in $\neg p$ for $p, r_{1}$ for $q_{1}$, and $r_{2}$ for $q_{2}$. This yields:

$$
\text { (B) } \mathrm{K} \vdash \diamond \square p \rightarrow \neg \bullet\left[\left(\diamond\left(\neg p \wedge r_{1}\right) \wedge \neg r_{1}\right) \vee\left(\diamond\left(\neg p \wedge r_{2}\right) \wedge \neg r_{2}\right)\right]
$$

Significantly, the consequent is a boxdot formula which is implied by the consequent of M. Since KM $\vdash$ M, from (A) and (B) we have:

$$
\begin{aligned}
\mathrm{KM} \vdash & \bullet\left[\left(\odot\left(p \wedge q_{1}\right) \wedge \neg q_{1}\right) \vee\left(\diamond\left(p \wedge q_{2}\right) \wedge \neg q_{2}\right)\right] \rightarrow \\
& \neg \odot\left[\left(\diamond\left(\neg p \wedge r_{1}\right) \wedge \neg r_{1}\right) \vee\left(\diamond\left(\neg p \wedge r_{2}\right) \wedge \neg r_{2}\right)\right]
\end{aligned}
$$

Call this theorem of KM: $\mathrm{e}^{\mathrm{M}}$ (' $\mathrm{e}$ ' for exterpolant). To complete our strategy with this example, we need to show $\mathrm{K} \psi \mathrm{e}^{\mathrm{M}}$. To see this, consider the following frame (arrows depicting the relation $R$ ), 


$$
\begin{aligned}
& \overline{1} \rightarrow \overline{2} \\
& \nearrow \text { X } \\
& 0 \rightarrow 1 \rightarrow 2
\end{aligned}
$$

And consider the model $M$ on the frame above with the valuation:

$$
V(p)=\{1,2\}, V\left(q_{1}\right)=\{1\}, V\left(q_{2}\right)=\{2\}, V\left(r_{1}\right)=\{\overline{1}\}, V\left(r_{2}\right)=\{\overline{2}\}
$$

The antecedent of $\mathrm{e}^{\mathrm{M}}$ is true at 0 . That is,

$$
M, 0 \vDash \odot\left[\left(\diamond\left(p \wedge q_{1}\right) \wedge \neg q_{1}\right) \vee\left(\diamond\left(p \wedge q_{2}\right) \wedge \neg q_{2}\right)\right]
$$

For consider the disjunction within the scope of $\square$ in the antecedent,

$$
\left(\diamond\left(p \wedge q_{1}\right) \wedge \neg q_{1}\right) \vee\left(\diamond\left(p \wedge q_{2}\right) \wedge \neg q_{2}\right)
$$

The left disjunct is true at 0 . For $M, 0 \vDash \neg q_{1}$, and since $0 R 1$ and $p \wedge q_{1}$ is true at $1, M, 0 \vDash \diamond\left(p \wedge q_{1}\right)$ (and thus $\left.M, 0 \vDash \diamond\left(p \wedge q_{1}\right)\right)$. Furthermore, the right disjunct is true at both 1 and $\overline{1}$. For $q_{2}$ is false at both 1 and $\overline{1}$, and since both 1 and $\overline{1}$ relate to 2 and $p \wedge q_{2}$ is true at $2, \diamond\left(p \wedge q_{2}\right)$ is true at both 1 and $\overline{1}$ (and thus $\odot\left(p \wedge q_{2}\right)$ is true at both 1 and $\left.\overline{1}\right)$. Thus the antecedent of $\mathrm{e}^{\mathrm{M}}$ is true at 0 . Yet the consequent of $\mathrm{e}^{\mathrm{M}}$ is false at 0 . That is,

$$
M, 0 \vDash \odot\left[\left(\diamond\left(\neg p \wedge r_{1}\right) \wedge \neg r_{1}\right) \vee\left(\diamond\left(\neg p \wedge r_{2}\right) \wedge \neg r_{2}\right)\right]
$$

For consider the disjunction,

$$
\left(\diamond\left(\neg p \wedge r_{1}\right) \wedge \neg r_{1}\right) \vee\left(\diamond\left(\neg p \wedge r_{2}\right) \wedge \neg r_{2}\right)
$$

The left disjunct is true at 0 . For $r_{1}$ fails at 0 , and since $0 R \overline{1}$ and $\neg p \wedge r_{1}$ is true at $\overline{1}, \diamond\left(\neg p \wedge r_{1}\right)$ is true at 0 . Furthermore, the right disjunct is true at 1 and $\overline{1}$. For $r_{2}$ fails at 1 and $\overline{1}$, and since both worlds relate to $\overline{2}$, and $\neg p \wedge r_{2}$ is true at $\overline{2}, \diamond\left(\neg p \wedge r_{2}\right)$ is true at both 1 and $\overline{1}$. Thus the consequent of $\mathrm{e}^{\mathrm{M}}$ is false at 0 . Thus,

$$
M, 0 \vDash \neg e^{\mathrm{M}}
$$

Thus $\mathrm{K} \nvdash \mathrm{e}^{\mathrm{M}}$. By Lemma 1.2, the Boxdot Conjecture holds for KM.

Where $\mathrm{M}^{\text {lmno }} \notin \mathrm{KT}$, our strategy is to find exterpolants for each $\mathrm{M}^{\text {lmno }}$ which are not theorems of K. Naturally, we use models to show these exterpolants are not theorems of K. Considering strategy, (it seems) there was a choice between complex models and simple exterpolants, or simple models and complex exterpolants. We go with the latter choice. We use a single frame for all models, and the models only differ in where $p$ is true.

$\mathrm{e}^{\mathrm{M}}$ is an exterpolant for $\mathrm{M}$, and the exterpolants we use for other $\mathrm{M}^{\text {lmno }}$ are variations on $\mathrm{e}^{\mathrm{M}}$. We used five propositional variables to construct $\mathrm{e}^{\mathrm{M}}$ (viz. $p, q_{1}, q_{2}, r_{1}$, and $r_{2}$ ). Due to our strategy, the larger the value of $l$, the larger the number of propositional variables we use to construct the antecedent of the exterpolant. Thus, consider $\mathrm{M}^{2100}$, i.e. $\square \square \diamond p \rightarrow p$. The following is a theorem of $\mathrm{K} \oplus \mathrm{M}^{2100}$ :

$$
\bullet \odot\left[\left(\diamond\left(p \wedge q_{1}\right) \wedge \neg q_{1}\right) \vee\left(\diamond\left(p \wedge q_{2}\right) \wedge \neg q_{2}\right) \vee\left(\diamond\left(p \wedge q_{3}\right) \wedge \neg q_{3}\right)\right] \rightarrow p
$$

By our method, this is the exterpolant we construct for $\mathrm{M}^{2100}$. 


\section{Preliminary Theorems}

The proofs of our first two Lemmas are left for the reader.

Lemma 3.1. If $\mathrm{K} \vdash \phi \rightarrow \psi$ then $\mathrm{K} \vdash \varpi^{z} \phi \rightarrow \varpi^{z} \psi$, all $z \geq 0$.

Lemma 3.2. $t \square^{z} \phi=\varpi^{z} t \phi$, all $z \geq 0$.

From Lemma 3.2 we have:

Corollary 3.3. $t \diamond^{z} \phi=\diamond^{z} t \phi$, all $z \geq 0$.

Lemma 3.4. $\mathrm{K} \vdash t \diamond^{z} \phi \leftrightarrow\left(\diamond^{z} t \phi \vee \diamond^{z-1} t \phi \vee \ldots \vee \diamond t \phi \vee t \phi\right)$, all $z \geq 0$.

Proof. This is Lemma 4.8 of [4].

Corollary 3.5. $\mathrm{K} \vdash \diamond^{z} t \phi \leftrightarrow\left(\diamond^{z} t \phi \vee \diamond^{z-1} t \phi\right)$, all $z \geq 1$.

Proof. Assume $z \geq 1$.

1) $\mathrm{K} \vdash \diamond^{z} t \phi \leftrightarrow\left(\diamond^{z} t \phi \vee \diamond^{z-1} t \phi \vee \ldots \vee t \phi\right)$, from Lem. 3.4 \& Cor. 3.3.

2) $\mathrm{K} \vdash \diamond^{z-1} t \phi \leftrightarrow\left(\diamond^{z-1} t \phi \vee \ldots \vee t \phi\right)$, from Lem. 3.4 \& Cor. 3.3.

3) $\mathrm{K} \vdash \diamond^{z} t \phi \leftrightarrow\left(\diamond^{z} t \phi \vee \diamond^{z-1} t \phi\right)$, from 1 and 2 , replacement.

The following is very useful.

Lemma 3.6. $\mathrm{K} \vdash\left(\diamond^{z}\left(p \wedge q_{g}\right) \wedge \odot^{z-1} \neg q_{g}\right) \rightarrow \diamond^{z} p$, for all $z \geq 1$.

Proof. Assume $z \geq 1$.

1) $\mathrm{K} \vdash\left(p \wedge q_{g}\right) \rightarrow p$.

2) $\mathrm{K} \vdash \diamond^{z}\left(p \wedge q_{g}\right) \rightarrow \diamond^{z} p$, from 1 .

3) $\mathrm{K} \vdash\left(\diamond^{z}\left(p \wedge q_{g}\right) \wedge \odot^{z-1} \neg q_{g}\right) \rightarrow \diamond^{z} p$, strengthening the antecedent of 2 .

4) $\mathrm{K} \vdash \neg q_{g} \rightarrow\left(\neg p \vee \neg q_{g}\right)$.

5) $\mathrm{K} \vdash \varpi^{z-1} \neg q_{g} \rightarrow \varpi^{z-1}\left(\neg p \vee \neg q_{g}\right)$, from 4 and Lemma 3.1.

6) $\mathrm{K} \vdash\left(\diamond^{z-1}\left(p \wedge q_{g}\right) \wedge \odot^{z-1} \neg q_{g}\right) \rightarrow \perp$,

from 5 using: if $\mathrm{K} \vdash \phi \rightarrow \psi$, then $\mathrm{K} \vdash(\neg \psi \wedge \phi) \rightarrow \perp$.

7) $\mathrm{K} \vdash\left(\diamond^{z-1}\left(p \wedge q_{g}\right) \wedge \otimes^{z-1} \neg q_{g}\right) \rightarrow \diamond^{z} p$, from 6 and $\mathrm{K} \vdash \perp \rightarrow \diamond^{z} p$.

8) $\mathrm{K} \vdash\left[\left(\diamond^{z}\left(p \wedge q_{g}\right) \wedge \odot^{z-1} \neg q_{g}\right) \vee\left(\diamond^{z-1}\left(p \wedge q_{g}\right) \wedge \odot^{z-1} \neg q_{g}\right)\right] \rightarrow \diamond^{z} p$,

from $3 \& 7$, if $\mathrm{K} \vdash \alpha \rightarrow \phi$ and $\mathrm{K} \vdash \beta \rightarrow \phi$, then $\mathrm{K} \vdash(\alpha \vee \beta) \rightarrow \phi$.

9) $\mathrm{K} \vdash\left[\left[\left(\diamond^{z}\left(p \wedge q_{g}\right) \vee \diamond^{z-1}\left(p \wedge q_{g}\right)\right] \wedge \bullet^{z-1} \neg q_{g}\right] \rightarrow \diamond^{z} p\right.$,

from 8 and $\mathrm{K} \vdash[[\alpha \vee \beta] \wedge \phi] \leftrightarrow[(\alpha \wedge \phi) \vee(\beta \wedge \phi)]$.

10) $\mathrm{K} \vdash \diamond^{z}\left(p \wedge q_{g}\right) \leftrightarrow\left[\diamond^{z}\left(p \wedge q_{g}\right) \vee \diamond^{z-1}\left(p \wedge q_{g}\right)\right]$, instance of Cor. 3.5.

11) $\mathrm{K} \vdash\left(\diamond^{z}\left(p \wedge q_{g}\right) \wedge \odot^{z-1} \neg q_{g}\right) \rightarrow \diamond^{z} p$, from 9 and 10, replacement.

Corollary 3.7. $\mathrm{K} \vdash\left(\diamond^{z}\left(p \wedge q_{g}\right) \wedge \odot^{z-1} \neg q_{g}\right) \rightarrow \diamond^{z}\left(p \wedge q_{g}\right)$, for all $z \geq 1$.

Proof. Assume $z \geq 1$.

1) $\mathrm{K} \vdash\left(\diamond^{z}\left(\left(p \wedge q_{g}\right) \wedge q_{g}\right) \wedge \odot^{z-1} \neg q_{g}\right) \rightarrow \diamond^{z}\left(p \wedge q_{g}\right)$, instance of Lem. 3.6.

2) $\mathrm{K} \vdash\left(\diamond^{z}\left(p \wedge q_{g}\right) \wedge \odot^{z-1} \neg q_{g}\right) \rightarrow \diamond^{z}\left(p \wedge q_{g}\right)$, from 1, idempotence.

Lemma 3.8. $\mathrm{K} \vdash \varpi^{n} p \rightarrow \square^{n} p$, all $n \geq 0$.

Proof. 1) K $\vdash t \square^{n} p \rightarrow \square^{n} p$, all $n \geq 0$, Lemma 4.4 of [4].

2) K $\vdash \odot^{n} p \rightarrow \square^{n} p$, all $n \geq 0$, from 1 and Lemma 3.2.

Australasian Journal of Logic (15:3) 2018 Article no. 2 
From Lemma 3.8 we have:

Corollary 3.9. $\mathrm{K} \vdash \diamond^{n} p \rightarrow \diamond^{n} p$, for all $n \geq 0$.

The following Theorem is useful for each case. Note how the number of propositional variables (and disjuncts) increases in the antecedent as $l$ increases. Thus, the number of propositional variables used is relevant to our strategy. Furthermore, which propositional variables used will also be relevant to our strategy (and is relative to the size of $m$ ). Exactly why this is strategic won't be clear until the next section. Suffice it to say, this minor complication will ultimately make it easier to uniformly show our exterpolants are not theorems of $\mathrm{K}$.

Theorem 3.10. For all $l \geq 0$ and $m \geq 1$,

$$
\mathrm{K} \vdash \bullet^{l}\left[\bigvee_{m \leq g \leq m+l}\left(\diamond^{m}\left(p \wedge q_{g}\right) \wedge \varpi^{m-1} \neg q_{g}\right)\right] \rightarrow \square^{l} \diamond^{m} p
$$

Proof. Assume $l \geq 0$ and $m \geq 1$.

$$
\text { 1) } \mathrm{K} \vdash\left[\bigvee_{m \leq g \leq m+l}\left(\diamond^{m}\left(p \wedge q_{g}\right) \wedge \bullet^{m-1} \neg q_{g}\right)\right] \rightarrow \diamond^{m} p
$$

using Lemma $3.6 l+1$ times together with repeated use of:

$$
\text { if } \mathrm{K} \vdash \alpha \rightarrow \phi \text { and } \mathrm{K} \vdash \beta \rightarrow \phi \text {, then } \mathrm{K} \vdash(\alpha \vee \beta) \rightarrow \phi \text {. }
$$

2) $\mathrm{K} \vdash \bullet^{l}\left[\bigvee_{m \leq g \leq m+l}\left(\diamond^{m}\left(p \wedge q_{g}\right) \wedge \bullet^{m-1} \neg q_{g}\right)\right] \rightarrow \bullet^{l} \diamond^{m} p$,

from 1 and Lemma 3.1.

3) $\mathrm{K} \vdash \nabla^{l} \diamond^{m} p \rightarrow \square^{l} \diamond^{m} p$, instance of Lemma 3.8.

4) $\mathrm{K} \vdash \bullet^{l}\left[\bigvee_{m \leq g \leq m+l}\left(\diamond^{m}\left(p \wedge q_{g}\right) \wedge \bullet^{m-1} \neg q_{g}\right)\right] \rightarrow \square^{l} \diamond^{m} p, 2$ and 3 .

We give three instances of Theorem 3.10 to illustrate how $m$ and $l$ determine which and how many propositional variables are used. If $l=0$ then our instance is Lemma 3.6 where $m=z=g$. If $l=2$ and $m=1$ we have,

$\mathrm{K} \vdash \bullet^{2}\left[\left(\diamond\left(p \wedge q_{1}\right) \wedge \neg q_{1}\right) \vee\left(\diamond\left(p \wedge q_{2}\right) \wedge \neg q_{2}\right) \vee\left(\diamond\left(p \wedge q_{3}\right) \wedge \neg q_{3}\right)\right] \rightarrow \square^{2} \diamond p$

And if $l=1$ and $m=4$ we have,

$$
\mathrm{K} \vdash \odot\left[\left(\diamond^{4}\left(p \wedge q_{4}\right) \wedge \odot^{3} \neg q_{4}\right) \vee\left(\diamond^{4}\left(p \wedge q_{5}\right) \wedge \odot^{3} \neg q_{5}\right)\right] \rightarrow \square \diamond^{4} p
$$

The following will be useful for our first case. Essentially we are taking the contraposition of Theorem 3.10 and replacing the occurrences of $q_{g}$ with $r_{i}$, and changing a number of other variables as well.

Corollary 3.11. For all $n \geq 0$ and $o \geq 1$,

$$
\mathrm{K} \vdash \diamond^{n} \square^{o} p \rightarrow \neg \bullet^{n}\left[\bigvee_{o \leq i \leq o+n}\left(\diamond^{o}\left(\neg p \wedge r_{i}\right) \wedge \bullet^{o-1} \neg r_{i}\right)\right]
$$

Proof. Take the contraposition of Theorem 3.10, then substitute $\neg p$ for $p$ and $r_{m}, \ldots, r_{m+l}$ for $q_{m}, \ldots, q_{m+l}$, and then change $l$ to $n, m$ to $o$, and $g$ to $i$. 


\section{The Frame and the Models}

We use one frame for all cases. The models only differ in where $p$ is true, and where $p$ is true depends on $m$. An illustration of the frame, $\dot{F}=\langle\dot{W}, \dot{R}\rangle$, is given below the following definition of the frame,

Definition 4.1. Let $\overline{\mathbb{N}}=\{\bar{z} \mid z \in \mathbb{N}\}$

Let $\dot{W}=\mathbb{N} \cup(\overline{\mathbb{N}}-\{\overline{0}\})$

Let $R=\{\langle x, y\rangle \mid x+1=y$ and $x, y \in \dot{W}\}$

Let $\bar{R}=\{\langle\bar{x}, \bar{y}\rangle \mid x+1=y$ and $\bar{x}, \bar{y} \in \dot{W}\}$

Let $R^{\lambda}=\{\langle x, \bar{y}\rangle \mid x+1=y$ and $x, \bar{y} \in \dot{W}\}$

Let $R^{\searrow}=\{\langle\bar{x}, y\rangle \mid x+1=y$ and $\bar{x}, y \in \dot{W}\}$

Let $\dot{R}=R \cup \bar{R} \cup R^{\rtimes} \cup R^{\searrow}$

Let $\dot{F}=\langle\dot{W}, \dot{R}\rangle$

Each world in $\dot{W}$ bears $\dot{R}$ to exactly two worlds. $\dot{F}=\langle\dot{W}, \dot{R}\rangle$ has a trellislike structure, exhibited as follows (arrows depicting $\dot{R}$ ),

$$
\begin{aligned}
& \overline{1} \rightarrow \overline{2} \rightarrow \overline{3} \rightarrow \overline{4} \rightarrow \overline{5} \rightarrow \overline{6} \rightarrow \ldots \\
& \text { オ オ オ } \\
& 0 \rightarrow 1 \rightarrow 2 \rightarrow 3 \rightarrow 4 \rightarrow 5 \rightarrow 6 \rightarrow \ldots
\end{aligned}
$$

We now define models for the frame. Models are defined relative to $m$. The intention is to falsify the relevant formula (the exterpolant) at 0 . Note that, for all of the following, the propositional variables in $\left\{q_{1}, q_{2}, \ldots\right\}$ are treated differently than the ones in $\left\{r_{1}, r_{2}, \ldots\right\}$. Officially, the complete set of propositional variables is,

$$
\{p\} \cup\left\{q_{1}, q_{2}, \ldots\right\} \cup\left\{r_{1}, r_{2}, \ldots\right\}
$$

Definition 4.2. Where $m \geq 1$,

Let $\dot{V}^{m}\left(q_{g}\right)=\{g\}$, for all $g \in \mathbb{N}-\{0\}$.

Let $\dot{V}^{m}\left(r_{i}\right)=\{\bar{i}\}$, for all $\bar{i} \in \overline{\mathbb{N}}-\{\overline{0}\}$.

Let $\dot{V}^{m}(p)=\{z \in \mathbb{N} \mid m \leq z\}$

Let $\dot{M}^{m}=\left\langle\dot{W}, \dot{R}, \dot{V}^{m}\right\rangle$.

Thus in $\dot{M}^{m}$, each $r_{i}$ is true at one and only one world (namely $\bar{i}$ ), and each $q_{g}$ is true at one and only one world (namely $g$ ). Thus the valuation of each $r_{i}$ and each $q_{g}$ is the same for all models (that is, regardless of the value of $m$ ). The valuation of $p$, in contrast, depends on $m$.

The following two Lemmas are simple and useful.

Lemma 4.3. For any $m \geq 1$, and any $\bar{x} \in \dot{W}$,

$$
\dot{M}^{m}, x \vDash \diamond \phi \text { iff } \dot{M}^{m}, \bar{x} \vDash \diamond \phi
$$

Proof. Assume $\dot{M}^{m}, x \vDash \diamond \phi$. Thus $\phi$ is true at either $x+1$ or $\overline{x+1}$, and $\bar{x}$ relates to both. Thus $\dot{M}^{m}, \bar{x} \vDash \diamond \phi$. The converse is similar. 
Lemma 4.4. For all $m, z \geq 1$, and all $w \in \dot{W}$,

$$
\text { If } \dot{M}^{m}, w \vDash \diamond^{z} q_{g} \text {, then } \dot{M}^{m}, w \vDash \otimes^{z-1} \neg q_{g}
$$

Proof. Assume $\dot{M}^{m}, w \vDash \diamond^{z} q_{g}(m, z \geq 1)$.

Since $g$ is the only world where $q_{g}$ is true, and $w$ is $z$ worlds away from $g, q_{g}$ fails at all $v$ and $\bar{v}$ such that $v<z$. Thus $\dot{M}^{m}, w \vDash \odot^{z-1} \neg q_{g}$.

Lemma 4.5. For all $m \geq 1$, and all $j \in \mathbb{N}$,

$$
\dot{M}^{m}, j \vDash \diamond^{m}\left(p \wedge q_{m+j}\right) \wedge \odot^{m-1} \neg q_{m+j}
$$

Proof. By induction on $j$. Where $j=0, \dot{M}^{m}, 0 \vDash \diamond^{m}\left(p \wedge q_{m}\right)$ because $m$ is $m$ worlds away from 0 , and $\dot{M}^{m}, m \vDash p \wedge q_{m}$. Thus $\dot{M}^{m}, 0 \vDash \diamond^{m}\left(p \wedge q_{m}\right)$.

Since $\dot{M}^{m}, 0 \vDash \diamond^{m} q_{m}$, it follows from Lemma 4.4 that $\dot{M}^{m}, 0 \vDash \odot^{m-1} \neg q_{m}$.

For the inductive step we assume the hypothesis holds for $c$ and show it holds for $c+1$ (instead of using the variable $n$, so that we may avoid any confusion with the $n$ in $\left.\mathrm{M}^{l m n o}\right)$. Assume

$$
\dot{M}^{m}, c \vDash \diamond^{m}\left(p \wedge q_{m+c}\right) \wedge \bullet^{m-1} \neg q_{m+c}
$$

By Cor. 3.7, $\dot{M}^{m}, c \vDash \diamond^{m}\left(p \wedge q_{m+c}\right)$.

Since $c$ is $m$ worlds away from $m+c, c$ must be $m+1$ worlds away from $m+c+1$ (by construction of the model). And since $p$ is true at $m+c, p$ is true at all numbers greater than $m+c$ (in $\mathbb{N})$. Thus, $\dot{M}^{m}, c \vDash \diamond^{m+1}\left(p \wedge q_{m+c+1}\right)$. Thus, there's some world $w, c \dot{R} w$ and $\dot{M}^{m}, w \vDash \diamond^{m}\left(p \wedge q_{m+c+1}\right)$. $w$ must be $c+1$ or $\overline{c+1}$. Either way, (since $m \geq 1$ ) by Lemma $4.3 \diamond^{m}\left(p \wedge q_{m+c+1}\right)$ is true at $c+1$. By Lem. 4.4, $\dot{M}^{m}, c+1 \vDash \diamond^{m}\left(p \wedge q_{m+c+1}\right) \wedge \odot^{m-1} \neg q_{m+c+1}$.

Theorem 4.6. For all $m \geq 1$ and all $l \geq 0$,

$$
\dot{M}^{m}, 0 \vDash \bullet^{l}\left[\bigvee_{m \leq g \leq m+l}\left(\diamond^{m}\left(p \wedge q_{g}\right) \wedge \bullet^{m-1} \neg q_{g}\right)\right]
$$

Proof. By induction on $l$. The base case, $l=0$, is

$$
\dot{M}^{m}, 0 \vDash \diamond^{m}\left(p \wedge q_{m}\right) \wedge \bullet^{m-1} \neg q_{m}
$$

This follows from Lemma 4.5, $j=0$.

As with the case of the inductive step of Lemma 4.5, we use the variable $c$ for the inductive step here (instead of the traditional $n$ ). Assume that

$$
\dot{M}^{m}, 0 \vDash \odot^{c}\left[\bigvee_{m \leq g \leq m+c}\left(\diamond^{m}\left(p \wedge q_{g}\right) \wedge \bullet^{m-1} \neg q_{g}\right)\right]
$$

To obtain a contradiction, assume that

$$
\dot{M}^{m}, 0 \vDash \diamond^{c+1} \neg\left[\bigvee_{m \leq g \leq m+c+1}\left(\diamond^{m}\left(p \wedge q_{g}\right) \wedge \odot^{m-1} \neg q_{g}\right)\right]
$$

Re-arranging the disjunction within the scope of $\odot^{c+1} \neg$ we have:

$$
\begin{aligned}
& \dot{M}^{m}, 0 \vDash \\
& \diamond^{c+1} \neg\left[\left(\diamond^{m}\left(p \wedge q_{m+c+1}\right) \wedge \odot^{m-1} \neg q_{m+c+1}\right) \vee\left(\bigvee_{m \leq g \leq m+c}\left(\diamond^{m}\left(p \wedge q_{g}\right) \wedge \otimes^{m-1} \neg q_{g}\right)\right)\right]
\end{aligned}
$$

By DeMorgan we have: 


$$
\begin{aligned}
& \dot{M}^{m}, 0 \vDash \\
& \diamond^{c+1}\left[\neg\left(\diamond^{m}\left(p \wedge q_{m+c+1}\right) \wedge \varpi^{m-1} \neg q_{m+c+1}\right) \wedge \neg\left(\bigvee_{m \leq g \leq m+c}\left(\diamond^{m}\left(p \wedge q_{g}\right) \wedge \varpi^{m-1} \neg q_{g}\right)\right)\right]
\end{aligned}
$$

Now, consider the sentence above, our induction hypothesis, and the following instance of Lemma 3.6: $\mathrm{K} \vdash\left(\diamond^{c+1}(\alpha \wedge \beta) \wedge \odot^{c} \neg \beta\right) \rightarrow \diamond^{c+1} \alpha$, where $\alpha$ is the sentence $\neg\left(\diamond^{m}\left(p \wedge q_{m+c+1}\right) \wedge \odot^{m-1} \neg q_{m+c+1}\right)$ and $\beta$ is the sentence $\neg\left(\bigvee_{m \leq g \leq m+c}\left(\diamond^{m}\left(p \wedge q_{g}\right) \wedge \odot^{m-1} \neg q_{g}\right)\right)$. By Modus Ponens we have:

$$
\dot{M}^{m}, 0 \vDash \diamond^{c+1} \neg\left(\diamond^{m}\left(p \wedge q_{m+c+1}\right) \wedge \odot^{m-1} \neg q_{m+c+1}\right)
$$

Thus, $\neg\left(\diamond^{m}\left(p \wedge q_{m+c+1}\right) \wedge \bullet^{m-1} \neg q_{m+c+1}\right)$ is true at either $c+1$ or $\overline{c+1}$, and from this we will derive a contradiction. By Lemma 4.5, we have:

$$
\dot{M}^{m}, c+1 \vDash \diamond^{m}\left(p \wedge q_{m+c+1}\right) \wedge \varpi^{m-1} \neg q_{m+c+1}
$$

Using Cor. 3.7, we have:

$$
\dot{M}^{m}, c+1 \vDash \diamond^{m}\left(p \wedge q_{m+c+1}\right)
$$

Since $m \geq 1$, by Lemma 4.3, we have:

$$
\dot{M}^{m}, \overline{c+1} \vDash \diamond^{m}\left(p \wedge q_{m+c+1}\right)
$$

Since $m \geq 1$, by Lemma 4.4 (and Cor. 3.9), we have:

$$
\dot{M}^{m}, \overline{c+1} \vDash \diamond^{m}\left(p \wedge q_{m+c+1}\right) \wedge \bullet^{m-1} \neg q_{m+c+1}
$$

Contradiction.

5. CASE 1: $l, m, o \geq 1 \& n \geq 0$.

Where $l, m, o \geq 1$ and $n \geq 0$, our first case is,

$$
\square^{l} \diamond^{m} p \rightarrow \diamond^{n} \square^{o} p
$$

No instance of this case is a theorem of KT. Consider a two world model where $a R a, a R b, b R a, b R b$, and $V(p)=\{a\}$. For all $m, o \geq 1, \diamond^{m} p$ and $\diamond^{o} \neg p$ are both valid in the model. Thus, for all $l, n \geq 0, \square^{l} \diamond^{m} p$ and $\square^{n} \diamond^{o} \neg p$ are valid in the model as well. Since the model is reflexive and KT is the logic of reflexive frames, no instance of this case is a theorem of KT.

Lemma 5.1. For all $l, m, o \geq 1$ and $n \geq 0$,

$$
\odot^{l}\left[\bigvee_{m \leq g \leq m+l}^{\mathrm{K} \oplus \mathrm{M}^{l m n o} \vdash}\left(\diamond^{m}\left(p \wedge q_{g}\right) \wedge \varpi^{m-1} \neg q_{g}\right)\right] \rightarrow \neg \varpi^{n}\left[\bigvee_{o \leq i \leq o+n}\left(\diamond^{o}\left(\neg p \wedge r_{i}\right) \wedge \varpi^{o-1} \neg r_{i}\right)\right]
$$

Proof. $\mathrm{K} \oplus \mathrm{M}^{l m n o} \vdash \square^{l} \diamond^{m} p \rightarrow \diamond^{n} \square^{o} p$, thus the result follows from Theorem 3.10 and Corollary 3.11.

Australasian Journal of Logic (15:3) 2018 Article no. 2 
We need to show that the sentence in the above Lemma fails at 0 in $\dot{M}^{m}$, and thus in particular that the consequent is false (by Theorem 4.6, given $m \geq 1$, the antecedent is true for all $l \geq 0$ at 0 in $\left.\dot{M}^{m}\right)$. Considering the basic similarity between the antecedent and the negation of the consequent, our proof of this is not much different than our proof of Theorem 4.6 (as well as the proof of Lemma 4.5). Thus the proofs we include are quicker.

Lemma 5.2. For all $m, z \geq 1$, and all $w \in \dot{W}$,

$$
\text { If } \dot{M}^{m}, w \vDash \diamond^{z} r_{i} \text {, then } \dot{M}^{m}, w \vDash \otimes^{z-1} \neg r_{i}
$$

Proof. $r_{i}$ is true at $\bar{i}$ and only $\bar{i}$, so the proof follows that of Lemma 4.4.

Lemma 5.3. For all $m, o \geq 1$, and all $j \in \mathbb{N}$,

$$
\dot{M}^{m}, j \vDash \diamond^{o}\left(\neg p \wedge r_{o+j}\right) \wedge \varpi^{o-1} \neg r_{o+j}
$$

Proof. The base case, $j=0$, is: $\dot{M}^{m}, 0 \vDash \diamond^{o}\left(\neg p \wedge r_{o}\right) \wedge \odot^{o-1} \neg r_{o}$. As $r_{o}$ is true at $\bar{o}$ and only $\bar{o}, \dot{M}^{m}, \bar{o} \not p$ (because $\dot{V}^{m}(p) \subseteq \mathbb{N}$ ), and $\bar{o}$ is $o$ worlds away from 0 , the base case is clear.

For the inductive step assume that $\dot{M}^{m}, c \vDash \odot^{o}\left(\neg p \wedge r_{o+c}\right) \wedge \odot^{o-1} \neg r_{o+c}$. Using Cor. 3.7, this implies $c$ is $o$ worlds away from $\overline{o+c}$, and thus $c$ is $o+1$ worlds away from $\overline{o+c+1}$, and since $p$ is false at all the $\bar{i}$ worlds, $\dot{M}^{m}, c \vDash \diamond^{o+1}\left(\neg p \wedge r_{o+c+1}\right)$. Thus at either $c+1$ or $\overline{c+1}, \diamond^{o}\left(\neg p \wedge r_{o+c+1}\right)$ is true. Either way, $\dot{M}^{m}, c+1 \vDash \diamond^{o}\left(\neg p \wedge r_{o+c+1}\right)$, by Lemma $4.3(o \geq 1)$. By Lemma $5.2, \dot{M}^{m}, c+1 \vDash \bullet^{o-1} \neg r_{o+c+1}$.

Lemma 5.4. Where $m, o \geq 1$ and $n \geq 0$,

$$
\dot{M}^{m}, 0 \vDash \odot^{n}\left[\bigvee_{o \leq i \leq o+n}\left(\diamond^{o}\left(\neg p \wedge r_{i}\right) \wedge \odot^{o-1} \neg r_{i}\right)\right]
$$

Proof. By induction on $n$. The base case $n=0$ is,

$$
\dot{M}^{m}, 0 \vDash \diamond^{o}\left(\neg p \wedge r_{o}\right) \wedge \odot^{o-1} \neg r_{o}
$$

This follows from Lemma $5.3(j=0)$. As mentioned, the proof of this Lemma is not much different than the proof of Theorem 4.6. The inductive step follows that of Theorem 4.6, using Lemma 5.3 in place of Lemma 4.5 and Lemma 5.2 in place of Lemma 4.4 where needed.

Theorem 5.5. For all $l, m, o \geq 1$ and $n \geq 0$,

$\bullet^{l}\left[\bigvee_{m \leq g \leq m+l}^{\mathrm{K}}\left(\diamond^{m}\left(p \wedge q_{g}\right) \wedge \bullet^{m-1} \neg q_{g}\right)\right] \rightarrow \neg \bullet^{n}\left[\bigvee_{o \leq i \leq o+n}\left(\diamond^{o}\left(\neg p \wedge r_{i}\right) \wedge \bullet^{o-1} \neg r_{i}\right)\right]$

Proof. By Theorem 4.6 and Lemma 5.4.

6. CASE 2: $l, m \geq 1, n \geq 0, o=0$.

Our second and final case is, where $l, m \geq 1$, and $n \geq 0$,

$$
\square^{l} \diamond^{m} p \rightarrow \diamond^{n} p
$$

In this case some instances are theorems of KT.

Australasian Journal of Logic (15:3) 2018 Article no. 2 
Lemma 6.1. Where $l, m, \geq 1, n \geq 0$,

$$
\mathrm{KT} \vdash \square^{l} \diamond^{m} p \rightarrow \diamond^{n} p \text { iff } m \leq n
$$

Proof. If $m \leq n$ then KT $\vdash \diamond^{m} p \rightarrow \diamond^{n} p$. And since KT $\vdash \square^{l} \diamond^{m} p \rightarrow \diamond^{m} p$,

$$
\mathrm{KT} \vdash \square^{l} \diamond^{m} p \rightarrow \diamond^{n} p
$$

Conversely, assume $m>n$, and define $R$ on the natural numbers with: $x R y$ iff $x=y$ or $x+1=y$. Let $V(p)=\{x \mid m \leq x\}$. Since $m>n, 0 \vDash \square^{n} \neg p$, and $0 \vDash \diamond^{m} p$. Moreover, $\diamond^{m} p$ is valid in the model, thus $0 \vDash \square^{l} \diamond^{m} p$, for all $l$. The model is reflexive, thus no such instance is a theorem of KT.

Thus, by this lemma, our second case is $l, m, \geq 1, n \geq 0, o=0$ and $m>n$.

Lemma 6.2. Where $l, m, \geq 1, n \geq 0, o=0$ and $m>n$,

$$
\mathrm{K} \oplus \mathrm{M}^{l m n o} \vdash \bullet^{l}\left[\bigvee_{m \leq g \leq m+l}\left(\diamond^{m}\left(p \wedge q_{g}\right) \wedge \odot^{m-1} \neg q_{g}\right)\right] \rightarrow \diamond^{n} p
$$

Proof. $\mathrm{K} \oplus \mathrm{M}^{l m n o} \vdash \square^{l} \diamond^{m} p \rightarrow \diamond^{n} p$, thus the result follows from Cor. 3.9 and Theorem 3.10.

Lemma 6.3. Where $m>n \geq 0$,

$$
\dot{M}^{m}, 0 \vDash \otimes^{n} \neg p
$$

Proof. By construction, for all $z<m, \dot{M}^{m}, z \vDash \neg p$ and $\dot{M}^{m}, \bar{z} \vDash \neg p$. Thus if $m>n, \dot{M}^{m}, 0 \vDash \otimes^{n} \neg p$.

Theorem 6.4. Where $l, m, \geq 1, n \geq 0$, and $m>n$,

$$
\mathrm{K} \Vdash \bullet^{l}\left[\bigvee_{m \leq g \leq m+l}\left(\diamond^{m}\left(p \wedge q_{g}\right) \wedge \odot^{m-1} \neg q_{g}\right)\right] \rightarrow \diamond^{n} p
$$

Proof. By Lemma 6.3 and Theorem 4.6

\section{Main Result}

The following Lemma is helpful in simplifying our cases.

Lemma 7.1. An instance of $\mathrm{M}^{l m n o}$ is an instance of $\mathrm{G}^{\text {hijk }}$ iff

$$
(l=0 \text { or } m=0) \text { and }(n=0 \text { or } o=0)
$$

Proof. If $(l=0$ or $m=0)$ and $(n=0$ or $o=0)$, then $\mathrm{M}^{l m n o}$ has no mixed modalities in either the antecedent or the consequent, thus it is an instance of $\mathrm{G}^{\text {hijk }}$.

Conversely, if $(l \geq 1$ and $m \geq 1)$ or $(n \geq 1$ and $o \geq 1)$, then either a box precedes a diamond in the antecedent, or a diamond precedes a box in the consequent. Either way, $\mathrm{M}^{l m n o}$ is not an instance of $\mathrm{G}^{\text {hijk }}$.

By Lemma 7.1, we can avoid redundancy with the work in [4].

Lemma 7.2. For all $\mathrm{M}^{l m n o} \notin \mathrm{KT}$,

$$
\begin{gathered}
\text { if }[(l, m, o \geq 1 \text { and } n \geq 0) \text { or }(l, m \geq 1 \text { and } n \geq 0 \text { and } o=0)], \\
\text { then }(\exists \psi)\left(\mathrm{K} \oplus \mathrm{M}^{l m n o} \vdash t \psi \text { and } \operatorname{KT} \psi \psi\right) .
\end{gathered}
$$

Australasian Journal of Logic (15:3) 2018 Article no. 2 
Proof. Assume $\mathrm{M}^{l m n o} \notin \mathrm{KT}$.

If $l, m, o \geq 1$ and $n \geq 0$, then this is case 1, from Section 5. By Lemma 5.1, Theorem 5.5 and Lemma 1.2, the desired conclusion follows.

If $l, m \geq 1$ and $n \geq 0$ and $o=0$, this is case 2, from Section 6. By assumption, $\mathrm{M}^{l m n o} \notin \mathrm{KT}$, thus we know by Lemma 6.1 that $m>n$. Let $\Psi$ be the theorem of $\mathrm{K} \oplus \mathrm{M}^{l m n o}$ in Lemma 6.2. By Theorem 6.4, when $m>n$, $\Psi$ is not a theorem of $\mathrm{K}$. By Lemma 1.2, the desired conclusion follows.

Theorem 7.3. For all $\mathrm{M}^{l m n o} \notin \mathrm{KT},(\exists \psi)\left(\mathrm{K} \oplus \mathrm{M}^{l m n o} \vdash t \psi\right.$ and $\left.\mathrm{KT} \nvdash \psi\right)$

Proof. Assume $\mathrm{M}^{l m n o} \notin \mathrm{KT}$. If $\mathrm{M}^{l m n o}$ is an instance of $\mathrm{G}^{\text {hijk }}$, the conclusion follows by Theorem 5.14 of [4].

If $\mathrm{M}^{l m n o}$ is not an instance of $\mathrm{G}^{h i j k}$, then by Lemma 7.1,

$(l \geq 1$ and $m \geq 1)$ or $(n \geq 1$ and $o \geq 1)$

Assume $l \geq 1$ and $m \geq 1$. Either $o=0$ or not. Either way, the conclusion follows by Lemma 7.2 .

Assume $n \geq 1$ and $o \geq 1$. Thus our axiom is: $\square^{l} \diamond^{m} p \rightarrow \diamond^{n} \square^{o} p$. By contraposition and substituting $\neg p$ for $p$, our axiom is equivalent to,

$$
\square^{n} \diamond^{o} p \rightarrow \diamond^{l} \square^{m} p
$$

Since $n, o \geq 1$, this case is isomorphic to the previous case.

Thus, for all $\mathrm{M}^{l m n o} \notin \mathrm{KT},(\exists \psi)\left(\mathrm{K} \oplus \mathrm{M}^{l m n o} \vdash t \psi \wedge \mathrm{KT} \nvdash \psi\right)$.

\section{Acknowledgments}

I thank Lloyd Humberstone for a fruitful email correspondence which led to this article, and for helpful remarks on an earlier draft. I thank an anonymous reviewer for a helpful, quick, and thorough review. I also thank my parents, John and Virginia, for everything.

\section{REFERENCES}

[1] G. Boolos, The Logic of Provability, 1993, Cambridge U. Press, Cambrdige, Mass.

[2] R. Goldblatt, I. Hodkinson, The McKinsey-Lemmon logic is barely canonical, The Australasian Journal of Logic, volume 3 (2007), pp. 1-19.

[3] R. French, L. Humberstone, Partial confirmation of a conjecture on the boxdot translation in modal logic, The Australasian Journal of Logic, volume 7 (2009), pp. 56-61.

[4] C. Steinsvold The boxdot conjecture and the language of essence and accident, The Australasian Journal of Logic, 10 (2011), pp. 18-35. 